\title{
ASOCIACIÓN ENTRE DOS LÍNEAS GENÉTICAS DE VACAS HOLSTEIN Y LA PÉRDIDA DE GESTACIÓN
}

\section{ASSOCIATION BETWEEN TWO GENETIC LINES OF HOLSTEIN COWS AND THE PREGNANCY LOSS}

Jennifer Gordillo Aldaz ${ }^{1}$, Iván Patricio Yánez-Ortiz², María Gabriela Arroyo Guarderas ${ }^{3}$, Ramiro Fernando Díaz Bolaños

\author{
${ }^{1}$ Instituto de Reproducción Animal Córdoba, Paraje Pozo del Tigre, Zona General Paz, Córdoba, Argentina. \\ ${ }^{2}$ Departament de Medicina i Cirurgia Animals, Universitat Autònoma de Barcelona, Travessera dels Turons, Bellaterra, \\ España. \\ ${ }^{3}$ Escuela de Medicina Veterinaria, Universidad San Francisco de Quito, Diego de Robles y Pampite, Cumbayá, Ecuador. \\ ${ }^{4}$ Instituto de Investigaciones en Biomedicina iBioMed, Universidad San Francisco de Quito, Diego de Robles y Pampite, \\ Cumbayá, Ecuador.
}

Email: rdiaz@usfq.edu.ec

\section{Información del artículo}

Tipo de artículo: Artículo original

Recibido:

$14 / 12 / 2020$

Aceptado:

$13 / 05 / 2021$

Licencia:

CC BY-NC-SA 4.0

Revista

ESPAMCIENCIA

12(1):1-7

DOI:

https://doi.org/10.51

260/revista_espamci encia.v12i1.236

\section{Resumen}

Con el objetivo de determinar la relación entre las líneas genéticas de Holstein-norteamericano (HNA) y Holstein-neozelandés (HNZ) con la pérdida de gestación entre los 30-60 días posinseminación artificial (dpIA) y, determinar la asociación entre el número de parto, número de servicios por preñez, intervalo entre partos e intervalo parto-preñez y la pérdida de gestación dentro de cada línea genética, se analizaron 403 lactaciones de 140 animales: 168 de 59 HNA y 235 de 81 HNZ. Se escogieron lactaciones de vacas gestantes (30dpIA). La pérdida de gestación se calculó con el resultado de su diagnóstico a los 60 dpIA. Se utilizó una regresión logística binaria para determinar la magnitud de la asociación. La pérdida de gestación se produjo en el 25,81\% (104/403) de las lactaciones analizadas: $27,98 \%$ (47/168) en vacas HNA y 24,26\% (57/235) en vacas HNZ. Las vacas HNA tuvieron 1,21 veces más probabilidades de sufrir pérdida de gestación que las vacas HNZ $(\mathrm{P}>0,05)$. Las HNA y HNZ multíparas tuvieron 2,52 y 2,37 veces más probabilidades de sufrir pérdida de gestación que las primíparas $(\mathrm{P}<0,05)$, respectivamente. Las vacas con dos o más servicios por preñez presentaron más probabilidades de sufrir pérdida de gestación $(2,48$ en HNA y 2,18 en HNZ; P<0,05). En conclusión, la línea genética de Holstein no se asoció significativamente con la pérdida de gestación entre los 30-60 dpIA. El número de parto en las vacas multíparas y el número de servicios por preñez ( $\geq 2$ ) fueron factores de riesgo para la pérdida de gestación en las dos líneas genéticas.

Palabras clave: Bovinos, fertilidad, lactación, número de parto, odds ratio, número de servicios por preñez.

\section{Abstract}

In order to determine the relationship between Holstein-North American (HNA) and Holstein-New Zealand (HNZ) genetic lines with pregnancy loss between 30-60 days post-artificial insemination (dpIA) and, to determine the association between the number of calving, number of services per pregnancy, calving interval and calving-pregnancy interval and the pregnancy loss within each genetic line, 403 lactations of 140 animals were analyzed: 168 of $59 \mathrm{HNA}$ and 235 of $81 \mathrm{HNZ}$. Lactations of pregnant cows (30dpIA) were chosen. Pregnancy loss was calculated with the result of its diagnosis at 60 dpIA. A binary logistic regression was used to determine the magnitude of the association. Pregnancy loss occurred in $25.81 \%(104 / 403)$ of the lactations analyzed: $27.98 \%(47 / 168)$ in HNA cows and $24.26 \%(57 / 235)$ in HNZ cows. HNA cows were 1.21 times more likely to suffer pregnancy loss than HNZ cows $(\mathrm{P}>0.05)$. Multiparous HNA and HNZ were 2.52 and 2.37 times more likely to suffer pregnancy loss than primiparous $(\mathrm{P}<0.05)$, respectively. Cows with two or more services per pregnancy were more likely to suffer pregnancy loss (2.48 in HNA and 2.18 in $\mathrm{HNZ}$; $\mathrm{P}<0.05)$. In conclusion, the Holstein genetic line was not significantly associated with pregnancy loss between 30-60 dpIA. The number of calving (multiparous) and the number of services per pregnancy $(\geq 2)$ were risk factors for pregnancy loss in the two genetic lines.

Keywords: Cattle, fertility, lactation, number of calving, odds ratio, number of services per pregnancy. 


\section{INTRODUCCIÓN}

La elección de la base genética para las zonas de mayor potencial lechero en la serranía ecuatoriana representa un problema práctico, ya que existen diversos criterios entre técnicos y productores sobre las preferencias para la selección de la línea genética ideal dentro de la raza Holstein (Lascano et al., 2017). Por un lado, está la línea de Holstein norteamericano (HNA) que ha sido seleccionada por su mayor producción de leche, tamaño corporal y angularidad en un sistema de producción basado en partos durante todo el año y altos niveles de suplementación con concentrados, con poco énfasis en características como la fertilidad (Horan et al., 2005; Miglior et al., 2005). Por otro lado, está la línea de Holstein neozelandés (HNZ) que ha sido seleccionada por su mayor rendimiento de sólidos lácteos y una mayor fertilidad y supervivencia en un sistema de producción basado en pastos (Harris y Kolver, 2001).

Dentro de la raza Holstein, el objetivo es incrementar la rentabilidad mediante la obtención mejores precios por la leche aumentando la calidad, especialmente de los contenidos sólidos de la leche (Taylor et al., 2016). Sin embargo, actualmente se reconoce a la eficiencia reproductiva como un objetivo primordial para incrementar la rentabilidad de los hatos lecheros, debido al impacto económico de la baja fertilidad en la producción de leche (Dezetter et al., 2017). Uno de los factores que produce una disminución de la fertilidad es la alta tasa de pérdida de gestación, principalmente si esta ocurre durante los primeros 45-60 días de gestación donde se han reportado pérdidas de hasta el 75\% (López-Gatius et al., 2004). En este periodo, la pérdida de gestación se ha caracterizado como una combinación entre una pérdida embrionaria tardía (42-50 días) y una pérdida fetal (>50 días) (Committee on Bovine Reproductive Nomenclature, 1972). Existen procesos fisiológicos importantes relacionados con la nutrición embrionaria/fetal, donde la placenta coriovitelina experimenta una involución para ser reemplazada funcionalmente por la placenta corioalantoidea, con lo cual se forman conexiones firmes entre los tejidos maternos y fetales en los placentomas, lo que permite una mayor difusión de nutrientes y gases directamente entre los sistemas circulatorios materno y fetal (Assis Neto et al., 2009). Los factores que pueden desencadenar la pérdida de gestación son la placentación inadecuada, la dificultad en la transición de la nutrición vitelina a la alantoidea, las alteraciones en la vascularización de la placenta y el subdesarrollo del embrión/feto (Wiltbank et al., 2016).

Los factores de riesgo relacionados con la pérdida de gestación más estudiados son: anomalías en los ovocitos, problemas en el reconocimiento materno de la gestación, anestro posparto prolongado, enfermedades infecciosas, producción de leche, baja condición corporal después del parto, balance energético negativo durante el periodo de transición, edad del animal, número de partos, días de lactancia al momento de la concepción, estrés calórico, la temporada, entre otros (Santos et al., 2004; Lee y Kim, 2007; Diskin et al., 2015). Además, existen otros factores que pueden provocar la pérdida de gestación como son factores genéticos (raza, familia, consanguinidad y grupos sanguíneos) y factores ambientales (nutrición, edad, infecciones, desequilibrio hormonal y ambiente uterino) (Boyd, 1965; Gábor et al., 2008; Diskin et al., 2012; Ma et al., 2019). En nuestro país no hay estudios realizados en los que la pérdida de gestación se atribuya al factor de raza o a la línea genética dentro de una raza. Los objetivos de este estudio fueron: 1) determinar la asociación entre las líneas genéticas (HNA y HNZ) y la pérdida de gestación entre los 30-60 días después de la inseminación artificial (IA), y 2) determinar la asociación entre el número de parto, número de servicios por preñez, intervalo entre partos e intervalo parto-preñez y la pérdida de gestación dentro de cada línea genética.

\section{MATERIALES Y MÉTODOS}

\section{Declaración ética}

El Comité de Bioética para el uso de animales en investigación y enseñanza de la Escuela de Medicina Veterinaria de la Universidad San Francisco de Quito, Ecuador, aprobó los métodos utilizados durante el presente trabajo, de acuerdo con los Estándares de Bienestar Animal y las recomendaciones de Reemplazo, Reducción y Refinamiento en la investigación animal, propuestas por Russell y Burch (1959). Número de aprobación: 2020-005.

\section{Recopilación de datos}

Los datos desde el 01 de enero de 2014 hasta el 31 de diciembre de 2018 se recopilaron de un hato lechero comercial ubicado en la parroquia Asunción de Alóag, cantón Mejía, provincia de Pichincha, Ecuador, a una altura de $2.762 \mathrm{msnm}$. La base de datos estuvo constituida por un total de 403 lactaciones correspondientes a 140 vacas Holstein, de las cuales 168 lactaciones fueron pertenecientes a 59 vacas HNA y 235 lactaciones a 81 vacas HNZ. Solo se eligieron las lactaciones de vacas que hayan tenido al menos un parto (primíparas), que hayan sido diagnosticadas como preñadas a los 30 días después de la IA mediante ultrasonografía transrectal y que tuvieran un periodo de espera voluntario de al menos 45 días desde el último parto hasta la primera IA. La información recogida del software ganadero (DairyLive ${ }^{\circledR}$, New Richmond, WI, USA) utilizado en el hato para cada animal fue: identificación individual, línea genética, número de parto, número de servicios por preñez, fecha de gestación, fecha de parto y diagnóstico de gestación a los 60 días después de la IA (preñada o vacía). Las líneas 
genéticas de las vacas estuvieron determinadas por el origen del material genético del padre (norteamericano o neozelandés). La tasa de pérdida de gestación entre los 3060 días después de la IA se calculó como el cociente entre el número de lactaciones de vacas vacías al día 60 y el número de lactaciones de vacas preñadas al día 30 , multiplicado por 100 .

\section{Análisis estadístico}

Los datos fueron analizados utilizando el procedimiento logístico (PROC LOGISTIC) del paquete estadístico SAS (2013), excluyendo del modelo estadístico factores de riesgo para la pérdida de gestación como la producción de leche y las enfermedades infecciosas. El diagnóstico de gestación a los 60 días después de la IA se ingresó en el modelo como una variable dicotómica dependiente (donde 1 denota preñada y 0 denota vacía). La línea genética (HNA y HNZ), el número de parto (primíparas y multíparas), el número de servicios por preñez (uno y dos o más), el intervalo entre partos (menos y más de 425 días) y el intervalo parto-preñez (menos y más de 134 días) en la pérdida de gestación se consideraron como variables independientes de clasificación en los análisis. Los coeficientes y la distribución aproximada de Chi-cuadrado de la estadística de Wald se utilizaron para calcular los odds ratios con intervalos de confianza (IC) del 95\%. Los valores de $\mathrm{P}<0,05$ se consideraron estadísticamente diferentes. Se tomó como referencia el procedimiento descrito por Garcia-Ispierto y López-Gatius (2019).

\section{RESULTADOS Y DISCUSIÓN}

De las 403 lactaciones analizadas, el 25,81\% (104/403) resultaron en pérdida de gestación entre los 30-60 días después de la IA, de las cuales el 27,98\% (47/403) correspondieron a la línea genética de HNA y el 24,26\% (57/403) a la línea genética de HNZ. El Cuadro 1 muestra que, de acuerdo a lactaciones, las vacas HNA tuvieron 1,21 veces más probabilidades de sufrir pérdida de gestación entre los 30-60 días después de la IA que las vacas HNZ ( $>>0,05)$. El intervalo de confianza del odds ratio osciló entre 0,77 y 1,90 , lo que establece que no existió una asociación estadísticamente significativa con un nivel de confianza del $95 \%$ entre las dos líneas genéticas de vacas Holstein y la pérdida de gestación. En otras palabras, la línea genética en esta raza no representa un factor de riesgo asociado a la pérdida de gestación.

Cuadro 1. Odds ratio de la pérdida de gestación entre los 30-60 días después de la inseminación artificial (IA) en el modelo de regresión logística binaria para las lactaciones de vacas Holstein Norteamericanas (HNA) y Holstein Neozelandesas (HNZ) $(n=403)$.

\begin{tabular}{clcccc}
\hline Factor & \multicolumn{1}{c}{$\begin{array}{c}\text { Línea } \\
\text { genética }\end{array}$} & $\begin{array}{c}\text { Pérdidas de } \\
\text { gestación }\end{array}$ & $\begin{array}{c}\text { Odds } \\
\text { ratio }\end{array}$ & $\begin{array}{c}\text { Intervalo de } \\
\text { confianza 95\% }\end{array}$ & Valor P \\
\hline $\begin{array}{c}\text { Pérdida de } \\
\text { gestación }\end{array}$ & HNA & $27,98 \%(47 / 168)$ & 1,21 & $0,77-1,90$ \\
\cline { 2 - 6 }
\end{tabular}

HNA: Holstein norteamericano, HNZ: Holstein neozelandés.

En el Cuadro 2 se muestran los odds ratios encontrados entre el número de parto, número de servicios por preñez, intervalo entre partos e intervalo parto-preñez y la pérdida de gestación dentro de cada línea genética. La tasa de pérdida de gestación entre los 30-60 días después del a IA fue significativamente mayor en las vacas multíparas ( $\geq 2$ partos) de ambas líneas genéticas $(33,94 \%$ en HNA y 29,22\% en HNZ) en comparación con las vacas primíparas (1 parto). El número de parto afectó significativamente la tasa de pérdida de gestación en las dos líneas genéticas, siendo las vacas HNA 2,52 y las vacas HNZ 2,37 veces más propensas a sufrir pérdida cuando han parido dos o más veces que las que han tenido un solo parto. El número de servicios por preñez presentó resultados similares para ambas líneas genéticas, encontrándose que las mayores pérdidas de gestación ocurrieron en vacas que han tenido dos o más servicios por preñez $(35,87 \%$ en HNA y $29,58 \%$ en HNZ) en relación a las que resultaron preñadas después de un solo servicio. Por lo tanto, estas vacas tuvieron más probabilidades de sufrir pérdida de gestación $(\mathrm{P}<0,05)$, siendo que los animales HNA con dos o más servicios por preñez tuvieron prácticamente 2,5 veces más probabilidades de perder la gestación, mientras que los animales HNZ con dos o más servicios por preñez presentaron más del doble $(2,18)$ de probabilidades de sufrir pérdida de gestación en comparación con las vacas que recibieron un solo servicio por preñez. El promedio del número de servicios por preñez fue de 1,96 para la línea genética de HNA y 1,97 la línea genética de HNZ. El intervalo entre partos y el intervalo parto-preñez no mostraron diferencia significativa en la pérdida de gestación en las dos líneas genéticas. Sin embargo, en ambos casos, la tasa de pérdida de gestación fue mayor en las vacas que presentaron un intervalo entre partos y un intervalo parto-preñez superior al promedio del hato (425 días y 134 días, respectivamente). 
Cuadro 2. Odds ratios de la pérdida de gestación entre los 30-60 días después de la inseminación artificial (IA) según número de parto, número de servicios por preñez, intervalo entre partos e intervalo parto-preñez dentro de cada línea genética de Holstein.

\begin{tabular}{|c|c|c|c|c|c|c|}
\hline Factor & $\begin{array}{c}\text { Línea } \\
\text { genética }\end{array}$ & Categoría & $\begin{array}{l}\text { Pérdidas de } \\
\text { gestación }\end{array}$ & $\begin{array}{l}\text { Odds } \\
\text { ratio }\end{array}$ & $\begin{array}{c}\text { Intervalo de } \\
\text { confianza } 95 \%\end{array}$ & Valor P \\
\hline \multirow{4}{*}{$\begin{array}{l}\text { Número de } \\
\text { parto }\end{array}$} & \multirow{2}{*}{$\begin{array}{l}\text { HNA } \\
(\mathrm{n}=168)\end{array}$} & Primíparas & $16,95 \%(10 / 59)$ & \multicolumn{3}{|c|}{ Referencia } \\
\hline & & Multíparas & $33,94 \%(37 / 109)$ & 2,52 & $1,15-5,53$ & 0,02 \\
\hline & \multirow{2}{*}{$\begin{array}{l}\mathrm{HNZ} \\
(\mathrm{n}=235)\end{array}$} & Primíparas & $14,81 \%(12 / 81)$ & \multicolumn{3}{|c|}{ Referencia } \\
\hline & & Multíparas & $29,22 \%(45 / 154)$ & 2,37 & $1,17-4,80$ & 0,02 \\
\hline \multirow{4}{*}{$\begin{array}{l}\text { Número de } \\
\text { servicios } \\
\text { por preñez }\end{array}$} & \multirow{2}{*}{$\begin{array}{l}\text { HNA } \\
(\mathrm{n}=168)\end{array}$} & 1 & $18,42 \%(14 / 76)$ & \multicolumn{3}{|c|}{ Referencia } \\
\hline & & $\geq 2$ & $35,87 \%(33 / 92)$ & 2,48 & $1,21-5,09$ & 0,01 \\
\hline & \multirow{2}{*}{$\begin{array}{l}\text { HNZ } \\
(n=235)\end{array}$} & 1 & $16,13 \%(15 / 93)$ & \multicolumn{3}{|c|}{ Referencia } \\
\hline & & $\geq 2$ & $29,58 \%(42 / 142)$ & 2,18 & $1,13-4,22$ & 0,02 \\
\hline \multirow{4}{*}{$\begin{array}{l}\text { Intervalo } \\
\text { entre partos }\end{array}$} & \multirow{2}{*}{$\begin{array}{l}\text { HNA } \\
(\mathrm{n}=82)\end{array}$} & $<425$ & $31,48 \%(17 / 54)$ & \multicolumn{3}{|c|}{ Referencia } \\
\hline & & $>425$ & $46,43 \%(13 / 28)$ & 1,89 & $0,74-4,82$ & 0,19 \\
\hline & \multirow{2}{*}{$\begin{array}{l}\text { HNZ } \\
(\mathrm{n}=122)\end{array}$} & $<425$ días & $33,33 \%(26 / 78)$ & \multicolumn{3}{|c|}{ Referencia } \\
\hline & & $>425$ días & $36,36 \%(16 / 44)$ & 1,14 & $0,53-2,48$ & 0,74 \\
\hline \multirow{4}{*}{$\begin{array}{l}\text { Intervalo } \\
\text { parto-preñez }\end{array}$} & \multirow{2}{*}{$\begin{array}{l}\text { HNA } \\
(\mathrm{n}=104)\end{array}$} & <134 días & $34,33 \%(23 / 67)$ & \multicolumn{3}{|c|}{ Referencia } \\
\hline & & $>134$ días & $35,14 \%(13 / 37)$ & 1,04 & $0,45-2,41$ & 0,93 \\
\hline & \multirow{2}{*}{$\begin{array}{l}\mathrm{HNZ} \\
(\mathrm{n}=149)\end{array}$} & $<134$ días & $26,04 \%(25 / 96)$ & \multicolumn{3}{|c|}{ Referencia } \\
\hline & & >134 días & $37,74 \%(20 / 53)$ & 1,72 & $0,84-3,53$ & 0,14 \\
\hline
\end{tabular}

HNA: Holstein Norte Americano, HNZ: Holstein Neozelandés.

La probabilidad de pérdida de gestación entre los 30-60 días después de la IA no difiere significativamente entre las dos líneas genéticas de Holstein y tampoco representan un factor de riesgo en este estudio (Cuadro 1). Esto es contrario a lo que ha sido estudiado previamente por Macdonald et al. (2008), en donde encontraron que una razón para que la tasa de preñez sea menor puede deberse a una mayor pérdida embrionaria o fetal. También, los hallazgos reportados por Dillon et al. (2006), muestran que las diferencias más bajas pronosticadas en las tasas de preñez en el primer servicio y el mayor número de servicios por vaca pueden atribuirse parcialmente a una mayor tasa de pérdida de gestación. Al aumentar el número de servicios por preñez, la probabilidad de pérdida de gestación aumenta, como se muestra en este estudio, lo que podría ser un factor de riesgo (Cuadro 2).

La tasa de pérdida de gestación entre los 30-60 días después de la IA obtenida en este estudio $(25,81 \%)$ es superior a lo reportado por Miranda et al. (2019), quienes en el mismo periodo encontraron que un $12 \%$ de vacas de un hato lechero comercial de la provincia de Pichincha perdieron la gestación, cuyas causa principal se asocia a un cierto grado de infección en la ubre (mastitis). En estudios realizados en otros países, se han registrado tasas de pérdida de gestación de 10,2\% hasta 13,5\% entre los 28-75 días de gestación (Fricke et al., 1998; Gümen et al., 2003; Rivera et al., 2004; Santos et al., 2009). Asimismo, dentro de cada línea genética, se obtuvieron tasas de pérdida de gestación (27,98\% en HNA y 24,26\% en HNZ) superiores a las descritas por McDougall et al. (2005), quienes reportan que las pérdidas de gestación en la línea genética Neozelandesa son inferiores a las registradas en el hemisferio norte para la línea genética Norteamericana, con tasas de pérdida de gestación de 6,4\% y de $12,8 \%$ entre los días 28-50 de gestación, respectivamente.

Desde mediados de la década de los años 1980, se estableció que la lactancia constituye un estado fisiológico que se asocia con una reducción de la eficiencia reproductiva (Badinga et al., 1985). En este sentido, las altas tasas de pérdida de gestación obtenidas en este estudio podrían atribuirse a los altos niveles de producción de leche que tiene el hato, el cual en promedio fue de 20,94 litros/vaca/día, siendo muy superior a la media nacional (6,65 litros/vaca/día) y alcanzando el doble de la media de la provincia de Pichincha (10,84 litros/vaca/día) (INEC, 2019), debido a que altos volúmenes de leche estarían asociados con un apoyo luteotrófico local inadecuado y una menor tasa de metabolización de la progesterona ovárica, la hormona responsable del mantenimiento de la gestación (Wiltbank et al., 2014; Miura, 2019). Walsh et al. (2011), encontraron que un alto volumen de producción de leche tiene un efecto negativo en la vaca, reflejado entre las 4-8 semanas después del parto, debido a una condición corporal baja, causada por un balance energético negativo, lo que retrasa la primera ovulación posparto. Desafortunadamente, la falta de información sobre la producción de leche en el momento de la pérdida de 
gestación no permitió una evaluación adecuada de esta afirmación.

De acuerdo con Thatcher y Santos (2013), otra posible razón para que se produzca pérdida de gestación se relaciona con los eventos metabólicos que ocurren después del parto. En el período posparto, las vacas lactantes entran en un balance energético negativo, en el que el consumo de energía no es capaz de sostener por completo la producción de leche. En este período, la glucosa producida se obtiene principalmente del torrente sanguíneo y es utilizada por la glándula mamaria para producir lactosa, lo que provoca una reducción en la disponibilidad de glucosa para un adecuado desarrollo fetal y placentario (Wathes et al., 2007).

El número de parto de las vacas se relacionó positivamente con la pérdida de gestación entre los 30-60 días después de la IA en las dos líneas genéticas en este estudio (Cuadro 2), siendo que la incidencia de pérdida de gestación fue significativamente mayor en las vacas que tienen más de dos partos (multíparas) en comparación con aquellas que solo han parido una vez (primíparas), lo que coindice con los resultados encontrados por otros autores que informaron que la pérdida de gestación aumentó después del tercer parto (Thurmond et al., 1990; Humblot, 2001; Lee y Kim, 2007). De esto se deduce que la edad de la vaca interfiere con el mantenimiento del embrión en el útero y, por tanto, con el mantenimiento de la gestación (Vanroose et al., 2000). Esto se debe probablemente a que los animales adultos tienen una menor actividad folicular con una menor calidad de ovocitos, lo que resulta en una menor capacidad de desarrollo de los embriones. Los datos del estudio realizado por Santos et al. (2009), demuestran que las vacas multíparas tienen una tasa de pérdida de preñez del $17,2 \%$ en comparación con las primíparas, que registran casi la mitad $(8,7 \%)$ entre los 30 58 días de gestación, siendo muy similares a los resultados obtenidos en este estudio en las dos líneas genéticas. También se ha demostrado que la calidad del endometrio se deteriora a medida que el animal envejece y que existe un nivel basal de mortalidad embrionaria, principalmente asociada a defectos genéticos que pueden ser heredados o adquiridos (Diskin y Morris, 2008).

El intervalo entre partos y el intervalo entre parto-preñez no fueron significativamente diferentes en la pérdida de gestación entre los 30-60 días después de la IA en las dos líneas genéticas de Holstein en este estudio (Cuadro 2), lo que también puede explicar por qué no hubo diferencia en el odds ratio entre las líneas genéticas (HNA y HNZ) y la pérdida de gestación. La pérdida de gestación contribuye a extender los intervalos entre parto y parto-preñez en todos los sistemas de manejo reproductivo (Bamber et al., 2009), aunque están asociados con la pérdida de gestación cuando se da una extensión de 80 días desde el primer servicio hasta la preñez y una extensión de 77 días en el intervalo entre partos sobre el promedio del hato (Fourichon et al., 2000).

Como se mencionó anteriormente, a pesar de que la raza se considera un factor de origen genético que puede estar relacionado con la pérdida de gestación, no existen estudios que apoyen esta teoría. A modo de ejemplo, Humblot y Denis (1986), informan que el retorno al estro de las vacas servidas es causado por un efecto del padre, pero sin evidencia genotípica o fenotípica. López-Gatius et al. (2002), encontraron que el material genético de un reproductor en particular utilizado para inseminación artificial en bovinos puede determinar que las vacas tengan 3,4 veces más probabilidades de sufrir pérdida de gestación entre los 38-90 días, siendo un factor de riesgo a considerar en la pérdida de gestación. No obstante, en el trabajo efectuado por Miguel et al. (2017) se encontraron diferencias significativas en función de la raza, al comparar las pérdidas de gestación en vacas Holstein argentinas versus vacas HNZ y versus vacas de la cruza entre HNZ con Jersey, que podrían ser comparables a lo de este estudio.

\section{CONCLUSIONES}

La línea genética de Holstein no se asoció estadísticamente con la pérdida de gestación entre 30-60 días después de la inseminación artificial (IA). El número de parto (multíparas) y el número de servicios por preñez $(\geq 2)$ fueron factores de ries go para la pérdida de gestación en vacas Holstein norteamericano (HNA) y en Holstein neozelandés (HNZ). Se necesitan más estudios que incluyan un mayor número de lactaciones y animales para corroborar los resultados obtenidos.

\section{LITERATURA CITADA}

Assis Neto, A. C., Pereira, F. T. V., Santos, T. C., Ambrosio, C. E., Leiser, R., y Miglino, M. A. 2009. Morpho-physical recording of bovine conceptus (Bos indicus) and placenta from days 20 to 70 of pregnancy. Reprod. Domest. Anim., 45(5): 760772.

Badinga, L., Collier, R. J., Thatcher, W. W., y Wilcox, C. J. 1985. Effects of climatic and management factors on conception rate of dairy cattle in subtropical environment. J. Dairy Sci., 68(1): 78-85.

Bamber, R. L., Shook, G. E., Wiltbank, M. C., Santos, J. E. P., y Fricke, P. M. 2009. Genetic parameters for anovulation and pregnancy loss in dairy cattle. J. Dairy Sci., 92(11): 5739-5753.

Boyd, H. 1965. Embryonic death in cattle, sheep and pigs. Vet. Bull. Weybridge, 35: 251-266. 
Committee on Bovine Reproductive Nomenclature. 1972. Recommendations for standardizing bovine reproductive terms. Cornell Vet., 62(2): 216-237.

Dezetter, C., Bareille, N., Billon, D., Côrtes, C., Lechartier, C., y Seegers, H. 2017. Changes in animal performance and profitability of Holstein dairy operations after introduction of crossbreeding with Montbéliarde, Normande, and Scandinavian Red. J. Dairy Sci., 100(10): 8239-8264.

Dillon, P., Berry, D. P., Evans, R. D., Buckley, F., y Horan, B. 2006. Consequences of genetic selection for increased milk production in European seasonal pasture based systems of milk production. Livest. Sci., 99(2-3): 141-158.

Diskin, M. G., y Morris, D. G. 2008. Embryonic and early foetal losses in cattle and other ruminants. Reprod. Domest. Anim., 43: 260-267.

Diskin, M. G., Parr, M. H., y Morris, D. G. 2012. Embryo death in cattle: an update. Reprod. Fertil. Dev., 24(1): 244-251.

Diskin, M. G., Waters, S. M., Parr, M. H., y Kenny, D. A. 2015. Pregnancy losses in cattle: potential for improvement. Reprod. Fertil. Dev., 28(2): 83-93.

Fourichon, C., Seegers, H., y Malher, X. 2000. Effect of disease on reproduction in the dairy cow: a metaanalysis. Theriogenology, 53(9): 1729-1759.

Fricke, P. M., Guenther, J. N., y Wiltbank, M. C. 1998. Efficacy of decreasing the dose of GnRH used in a protocol for synchronization of ovulation and timed AI in lactating dairy cows. Theriogenology, 50(8): 1275-1284.

Gábor, G., Tóth, F., Ózsvári, L., Abonyi-Tóth, Z., y Sasser, R. G. 2008. Factors influencing pregnancy rate and late embryonic loss in dairy cattle. Reprod. Domest. Anim., 43(1): 53-58.

Garcia-Ispierto, I., y López-Gatius, F. 2019. Abortion in dairy cattle with advanced twin pregnancies: incidence and timing. Reprod. Domest. Anim., 54(Suppl. 4): 50-53.

Gümen, A., Guenther, J. N., y Wiltbank, M. C. 2003. Follicular size and response to ovsynch versus detection of estrus in anovular and ovular lactating dairy cows. J. Dairy Sci., 86(10): 3184-3194.

Harris, B. L., y Kolver, E. S. 2001. Review of Holsteinization on intensive pastoral dairy farming in New Zealand. J. Dairy Sci., 84(E. Suppl.): E56-
E61.

Horan, B., Dillon, P., Faverdin, P., Delaby, L., Buckley, F., y Rath, M. 2005. The interaction of strain of Holstein-Friesian cows and pasture-based feed systems on milk yield, body weight, and body condition score. J. Dairy Sci., 88(3): 1231-1243.

Humblot, P. 2001. Use of pregnancy specific proteins and progesterone assays to monitor pregnancy and determine the timing, frequencies and sources of embryonic mortality in ruminants. Theriogenology, 56(9): 1417-1433.

Humblot, P., y Denis, J. B. 1986. Sire effects on cow fertility and late embryonic mortality in the montbeliard breed. Livest. Prod. Sci., 14(2): 139148.

INEC (Instituto Nacional de Estadísticas y Censos, EC). 2019. Encuesta de Superficie y Producción Agropecuaria Continua (ESPAC) 2019. Disponible en:

https://www .ecuadorencifras.gob.ec/documentos/w eb-inec/Estadisticas_agropecuarias/espac/espac2019/Presentacion de los principales resultados ESPAC 2019.pdf

Lascano, P., Arcos, C., Guevara, R., Torres, C., Guevara, G., Serpa, G., Curbelo, R., y Pedraza, R. 2017. Respuesta productiva de vacas lecheras neozelandesas sometidas a pastoreo rotacional en el trópico alto del norte de Ecuador. Maskana, 8: 153155.

Lee, J. I., y Kim, I. H. 2007. Pregnancy loss in dairy cows: the contributing factors, the effects on reproductive performance and the economic impact. J. Vet. Sci., 8(3): 283-288.

López-Gatius, F., Santolaria, P., Yániz, J. L., Garbayo, J. M., y Hunter, R. H. F. 2004. Timing of early foetal loss for single and twin pregnancies in dairy cattle. Reprod. Domest. Anim., 39(6): 429-433.

López-Gatius, F., Santolaria, P., Yániz, J., Rutllant, J., y López-Béjar, M. 2002. Factors affecting pregnancy loss from gestation day 38 to 90 in lactating dairy cows from a single herd. Theriogenology, 57(4): 1251-1261.

Ma, L., Cole, J. B., Da, Y., y VanRaden, P. M. 2019. Symposium review: genetics, genome-wide association study, and genetic improvement of dairy fertility traits. J. Dairy Sci., 102(4): 3735-3743.

Macdonald, K. A., Verkerk, G. A., Thorrold, B. S., Pryce, 
J. E., Penno, J. W., McNaughton, L. R., Burton, L. J., Lancaster, J. A. S., Williamson, J. H., y Holmes, C. W. 2008. A comparison of three strains of Holstein-Friesian grazed on pasture and managed under different feed allowances. J. Dairy Sci., 91(4): 1693-1707.

McDougall, S., Rhodes, F. M., y Verkerk, G. A. 2005. Pregnancy loss in dairy cattle in the Waikato region of New Zealand. N. Z. Vet. J., 53(5): 279-287.

Miglior, F., Muir, B. L., y Van Doormaal, B. J. 2005. Selection indices in Holstein cattle of various countries. J. Dairy Sci., 88(3): 1255-1263.

Miguel, B., Casaro, G., y Daglio, M. C. 2017. Cuantificación de pérdidas de gestación entre los días 35-100 en vacas de tambo de la Cuenca Mar y Sierras. Tesis. Ciencias Veterinarias. UNCPBA. Tandil-Buenos Aires. AR. p 14-17.

Miranda, S., Albuja, C., y Tríbulo, H. 2019. Asociación entre la mastitis subclínica con la pérdida temprana de gestación en un hato de vacas lecheras. La Granja Rev. Ciencias La Vida, 30(2): 48-56.

Miura, R. 2019. Physiological characteristics and effects on fertility of the first follicular wave dominant follicle in cattle. J. Reprod. Dev., 65(4): 289-295.

Rivera, H., López, H., y Fricke, P. M. 2004. Fertility of Holstein dairy heifers after synchronization of ovulation and timed AI or AI after removed tail chalk. J. Dairy Sci., 87(7): 2051-2061.

Russell, W. M. S., y Burch, R. L. 1959. The principles of humane experimental technique. London, UK: Methuen.

SAS (Statistical Analysis System). 2013. V 9.4, SAS Institute Inc., Cary, NC, USA, Obtenido de: https://support.sas.com/software/94/

Santos, J. E. P., Rutigliano, H. M., y Sá Filho, M. F. 2009. Risk factors for resumption of postpartum estrous cycles and embryonic survival in lactating dairy cows. Anim. Reprod. Sci., 110(3-4): 207-221.
Santos, J. E. P., Thatcher, W. W., Chebel, R. C., Cerri, R. L. A., y Galvão, K. N. 2004. The effect of embryonic death rates in cattle on the efficacy of estrus synchronization programs. Anim. Reprod. Sci., 8283: 513-535.

Taylor, J. F., Taylor, K. H., y Decker, J. E. 2016. Holsteins are the genomic selection poster cows. Proc. Natl. Acad. Sci., 113(28): 7690-7692.

Thatcher, W. W., y Santos, J. E. P. 2013. Nuevas estrategias para prevenir la mortalidad embrionaria en el ganado lechero. Taurus, 57: 4-25.

Thurmond, M. C., Picanso, J. P., y Jameson, C. M. 1990. Considerations for use of descriptive epidemiology to investigate fetal loss in dairy cows. J. Am. Vet. Med. Assoc., 197(10): 1305-1312.

Vanroose, G., de Kruif, A., y Van Soom, A. 2000. Embryonic mortality and embryo-pathogen interactions. Anim. Reprod. Sci., 60-61: 131-143.

Walsh, S. W., Williams, E. J., y Evans, A. C. O. 2011. A review of the causes of poor fertility in high milk producing dairy cows. Anim. Reprod. Sci., 123(34): $127-138$.

Wathes, D. C., Fenwick, M., Cheng, Z., Bourne, N., Llewellyn, S., Morris, D. G., Kenny, D., Murphy, J., y Fitzpatrick, R. 2007. Influence of negative energy balance on cyclicity and fertility in the high producing dairy cow. Theriogenology, 68(Suppl. 1): S232-S241.

Wiltbank, M. C., Baez, G. M., Garcia-Guerra, A., Toledo, M. Z., Monteiro, P. L. J., Melo, L. F., Ochoa, J. C., Santos, J. E. P., y Sartori, R. 2016. Pivotal periods for pregnancy loss during the first trimester of gestation in lactating dairy cows. Theriogenology, 86(1): 239-253.

Wiltbank, M. C., Souza, A. H., Carvalho, P. D., Cunha, A. P., Giordano, J. O., Fricke, P. M., Baez, G. M., y Diskin, M. G. 2014. Physiological and practical effects of progesterone on reproduction in dairy cattle. Animal, 8(s1): 70-81. 